\title{
Acupressure and Anxiety in Cancer Patients
}

\author{
Ali Beikmoradi ${ }^{1}$; Fatemeh Najafi ${ }^{2,{ }^{*}}$; Ghodratallah Roshanaei ${ }^{3}$; Zahra Pour Esmaeil ${ }^{4}$; \\ Mahnaz Khatibian ${ }^{5}$; Alireza Ahmadi ${ }^{6}$ \\ ${ }^{1}$ Chronic Disease Home Care Research Center, Hamadan University of Medical Sciences, Hamadan, IR Iran \\ ${ }^{2}$ Department of Nursing, Faculty of Nursing and Midwifery, Hamadan University of Medical Sciences, Hamadan, IR Iran \\ 3 Reparch Center for Modeling of Non-Communicable Diseases, Hamadan University of Medical Sciences, Hamadan, IR Iran \\ ${ }_{4}^{4}$ Department of Nursing, Faculty of Nursing and Midwifery, Shahid Beheshti University of Medical Sciences, Tehran, IR Iran \\ ${ }_{5}$ Repartment of Nursing, Faculty of Nursing and Midwifery, Shahid Beheshti University of Medical Sciences, \\ ${ }_{6}^{6}$ Research Center for Maternal and Child Care, Hamadan University of Medical Sciences, Hamadan, IR Iran \\ ${ }^{*}$ Corresponding Author: Fatemeh Najafi, Department of Nursing, Faculty of Nursing and Midwifery, Hamadan University of Medical Sciences, Hamadan, IR Iran. Tel: +98-9127123830, \\ E-mail: fatima_naj_90@yahoo.com
}

Received: December 8, 2014; Revised: January 4, 2015; Accepted: February 23, 2015

\begin{abstract}
Background: Anxiety has negative effects on mental and physical performance, quality of life, duration of hospitalization, and even on the treatment of patients with cancer.

Objectives: Today acupressure is widely used to treat anxiety. Thus, the present study aimed to investigate the effects of acupressure on anxiety in patients with cancer.

Patients and Methods: A double-blind randomized clinical trial was conducted on 85 patients hospitalized with 3 groups including acupressure group $(n=27)$, sham group $(n=28)$, and control group $(n=30)$ in the hematologic ward of Shahid Beheshti Hospital of Hamadan, Iran, in 2013. The sampling permuted-block randomization with triple block was used. The anxiety of the patients in the experimental, sham, and control groups were measured with Spielberger's State-Trait Anxiety Inventory (STAI). Then, real acupressure was performed in the experimental group and fake acupressure in the sham group, and only routine care was provided for the control group. Anxiety of the patients was also assessed at 5 and 10 days after the intervention. Statistical analysis of the data was performed by SPSS software using repeated measures analysis of variance (ANOVA) and post hoc least significant difference (LSD) test.

Results: According to the findings, the mean level of anxiety before the intervention between groups were matched $(\mathrm{P}>0.05)$. Acupressure had a significant influence on the anxiety in the experimental group at $5(45.30 \pm 7.14)$ and 10 days $(43.48 \pm 6.82)$ after the intervention $(P<$ 0.05). However, it did not have a significant impact on their covert anxiety $\left(45.48 \pm 7.92\right.$ at $5^{\text {th }}$ day vs $45.63 \pm 8.08$ at 10 th day, $\left.P>0.05\right)$. No significant differences were observed in the fake points regarding overt and covert anxiety of patients in the sham group (overt anxiety; $47.57 \pm 7.85$ at $5^{\text {th }}$ day vs. $46.71 \pm 7.32$ at $10^{\text {th }}$ day, $\mathrm{P}>0.05$ ) (covert anxiety; $47.96 \pm 6.33$ at $5^{\text {th }}$ day vs. $46.89 \pm 6.94$ at $10^{\text {th }}$ day, $\mathrm{P}>0.05$ ). Moreover, the routine care provided for the control group did not have any effect on the overt and covert anxiety of the patients $(\mathrm{P}>0.05)$.

Conclusions: Acupressure is recommended as a complementary therapy to reduce anxiety in patients with cancer because of its low cost, safety, and simplicity.
\end{abstract}

Keywords: Neoplasms; Anxiety; Acupressure

\section{Background}

Cancer risk factors and its mortality rate are rising in today's world. More than 10 million new cases of cancer are reported worldwide each year (1). Today, cancer diagnosis is not equivalent to imminent death, however, many studies show the emergence of emotional and anxiety problems in these patients after cancer diagnosis (2). According to the studies, between $6 \%$ and $34 \%$ of patients with cancer suffer from anxiety (3-5). Another study reported an incidence of about $49 \%(3,6)$. In the early stages of cancer treatment, $80 \%$ of the patients suffer from severe anxiety (7). Through lowering patient's independence and ability to act in the family and society, Cancer causes lack of competence and confidence, low self-esteem, and anxiety (8). Anxiety is the most common reaction to stress and any threat caused by exposure to unfamiliar situations or risk of death due to cancer (9). Anxiety may be caused due to a variety of reasons such as response to cancer diagnosis, long-term treatments, side effects of treatment, poor treatment adherence, frequent hospitalization, changes in the normal pattern of life, decreased quality of life, possible disfigurement, extreme pain, financial and social issues, dependence on others, family problems, and death or dying process $(10,11)$. Psychological care is an important part of health provision, immediately after cancer diagnosis until the end of the treatment and follows up (12). Treatment of anxiety in patients with cancer can improve their emotional, social, and cognitive functions, as well as their interpersonal relationships with family, friends, and formal or informal caregivers (3). Most often, no attention is paid to anxiety in patients with can- 
Beikmoradi A et al.

cer; however, pharmacological and nonpharmacological methods are used to treat anxiety of these patients (13). Administration of benzodiazepine to treat anxiety is common, and alone or with other sedatives, it usually causes respiratory depression or long-term sedation (14). Other treatment of anxiety is cognitive behavioral therapy that is not widely available. Consequently, other treatment options are sought by patients (15).

Today, using complementary medicine as a nonpharmacological approach in conjunction with nursing care and common treatments as clinical expertise and competence are emphasized (16). Among the existing complementary medicine techniques, acupuncture, and acupressure are most commonly used in patients with cancer (17). Acupressure is a therapy used to manage various symptoms (18). Acupressure is rooted in acupuncture, and the hands or elbows are used to apply physical pressure to acupoints for treatment (18-20). Acupressure is based on the belief that vital energy (Chi/Qi) in the body is circulating through 12 main meridians (21). Approximately 365 acupoints have been specified on these channels, and each of these points affects the performance of a specific body part (22). Acupressure is based on the belief that problems, dysfunctions, and diseases are caused by an imbalance in the flow of vital energy in the body. Thus, by stimulating these specific points, the flow of vital energy can be balanced, and the problems, dysfunctions, or diseases can be treated (15). In addition, in medical science there is a theory that acupressure, by adjusting the concentration of neurotransmitters and reducing the concentration of 5-hydroxytryptamine and adrenocorticotropic hormone in neural pathways, reduces anxiety (23). Acupressure promotes relaxation, relieves dyspnea, and enhances immunity (20). Nurses have intimate and long-term relationships with patients, and can apply acupressure, as part of their nursing care, to reduce and treat patients' illnesses (24). Several studies have been performed on the effect of acupressure in treating and relieving anxiety before abdominal surgery, or transferring to the hospital (25-28).

\section{Objectives}

Because of the increased risk of cancer in Iranian people, the prevalence of anxiety in patients with cancer, noninvasive and simple use of acupressure, the importance of assessment, treatment, and relieving anxiety, as well as the limited number of studies in this respect, the present study aimed to evaluate the effectiveness of acupressure on the anxiety of patients with cancer $(25,29)$.

\section{Patients and Methods}

This was a double-blind clinical trial with 3 groups on 85 patients with cancer hospitalized at the hematology ward of Shahid Beheshti Hospital of Hamadan, Iran, in 2013. This study was conducted in 2013 at the hematology ward of Shahid Beheshti Hospital of Hamadan, a general governmental hospital with 7 wards and 180 bed. All patients voluntarily participated in this study and a written informed consent was obtained from each of them before the study. All the study procedures were approved by the Research Ethics Committee of Hamadan University of Medical Sciences and the protocol was registered at the Iranian Registry of Clinical Trials (IRCT201301219759N2).

Hospitalized patients with cancer were enrolled in this study based on the following criteria: diagnosis of cancer (any type of cancer) was done at least 1 month prior to the study, having full awareness, being 18 to 65 years old, lack of amputation or wounds at acupoints, and having anxiety based on the State-Trait Anxiety Inventory (STAI) (scores greater than 30). Patients were excluded if they had less than $50000 / \mathrm{mL}$ platelets, became pregnant, or developed consciousness disorders. Study details were described for the participants and a written informed consent was obtained from them before the intervention.

\subsection{Validity and Reliability}

The data collection tool was a questionnaire that consisted of 3 parts. The first part included demographic questions about age, gender, occupation, marital status, education level, income, type of illness, and additional information about the disease stage and the final examinations in the patients' medical records. Second and third sections included the STAI with a Cronbach $\alpha$ values of 0.93 and 0.91 (32). To determine the validity of STAI, content validity was used. The questionnaire was given to 10 faculty members of Hamadan University of Medical Sciences to be revised. To determine the reliability of the questionnaire, Cronbach $\alpha$ test was used. The reliability of STAI questionnaire was 0.88 .

STAI comprised 20 items based on the Likert scale $(1=$ very low, $2=$ low, $3=$ high, and $4=$ very high) and showed the feelings of the participants at the time of answering. The Trait Anxiety Inventory included 20 items based on the Likert scale ( $1=$ almost never, $2=$ occasionally, $3=$ most of the time, and $4=$ almost always) and measured the general and ordinary feelings of the participants at any time. STAI scores ranged between 20 and 80 with higher scores indicating greater anxiety. Cut-off score of 39-40 has been suggested for the detection of clinically significant symptoms of overt anxiety (30). The authors of the present study also used this score for trait anxiety in their interpretations. However, in another study, higher cut-off score of 54-55 was recommended for older adults (31).

\subsection{Interventions}

Patients in the acupressure group received the intervention in 25- to 30-minute sessions (one session per day for 10 days) by the researcher, who pressed his or her thumb at each acupoint alternately for 2 minutes. Based on the resources and the assistant acupressure expert's opinion, 9 acupoints were determined. These points included 5 two-way points of Shen men (located on the upper wall 

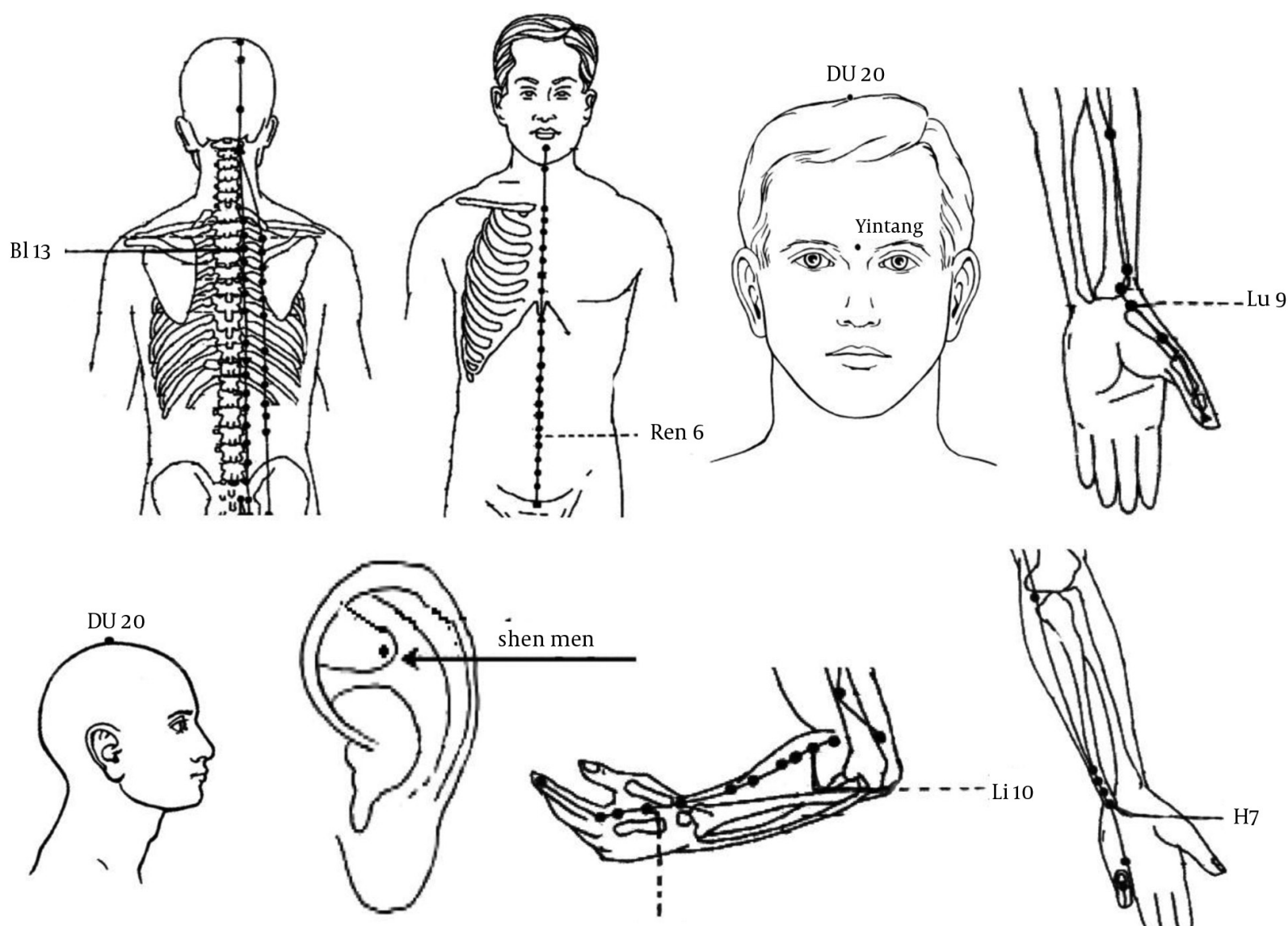

shen men
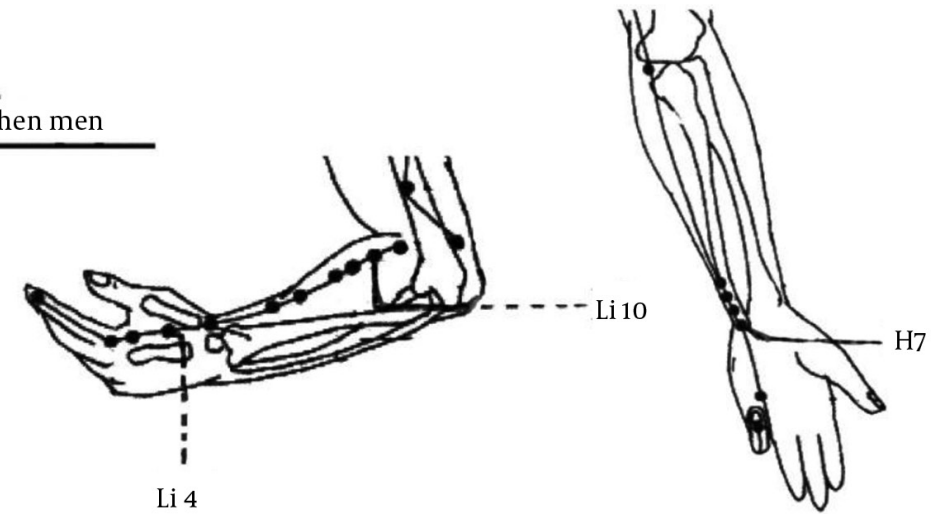

Figure 1. Selected Acupoint in Acupressure Group

of the ear cavity) (32), LI4 (middle of the bisector of the angle between the first and second metacarpal), LI10 (two fingers above the elbow joint towards radial), $\mathrm{H} 7$ (on the lateral wrist, radial towards the tendon of flexor carpi ulnaris), Lu9 (on the edge of the radial carpal joint outside the radial artery), and 4 one-way points of DU20 (intersection of the head middle line and its lateral line), Ren6 (2.5 $\mathrm{cm}$ below the navel), Yin tang (point between the two eyebrows), and UB13 (2.5 cm of the right side of T3) (33) (Figure 1). Patients in the sham group received acupressure during 25- to 30-minute sessions (one session per day for 10 days) by the researcher, using his thumb on the fake acupoints alternately for 2 minutes. The fake acupoints in the sham group consisted of 9 points with a distance of $2 \mathrm{~cm}$ from the points of the acupressure group. Patients in the control group only received routine care. Patients in the acupressure group and sham group completed the STAI before and after the interventions of sessions 5 and 10. The control group also completed the questionnaire before and after days 5 and 10. The expected outcomes were state and trait anxiety, which were assessed as selfreports by the patients. State and trait anxiety were assessed before and after the intervention using STAI by the research assistant through asking questions.

\subsection{Sample Size}

Based on the results of the study by Tsay et al. (34) on the effects of acupressure on fatigue in patients with chronic renal failure, and considering $\alpha=0.05$ and test power of $90 \%$, the required number of subjects for each group was calculated at 25 . Considering a $15 \%$ loss, this number was estimated at 30 people in each group and a total of 90 participants. The method and formula that were used for calculation of the sample size in the analysis of variance (ANOVA) method, was based on $\alpha$ and $\beta$ and can be found in Norman and Streiner (35).

$$
\begin{gathered}
\delta=\mu_{\max }-\mu_{\text {min }}=2 \\
S=1.8 \\
d=\frac{\delta}{S}=\frac{2}{1.8}=1.11
\end{gathered}
$$




$$
f=\frac{d}{2} \sqrt{\frac{k+1}{3(k+1)}}=0.45
$$

Where, s, d, and f denote standard deviation, effect size, and ANOVA effect size, respectively.

\subsection{Randomizations}

Eligible patients were randomly assigned to three groups of acupressure, sham, and control using permuted-block randomization with triple block. Then, acupressure, sham, and control groups were named A, B, and C, respectively. Therefore, for every 3 patients, one of the 6 possible blocks of $\mathrm{ABC}, \mathrm{ACB}, \mathrm{BAC}, \mathrm{BCA}, \mathrm{CAB}$, and $\mathrm{CBA}$ was selected randomly and each patient was assigned to the respective group. (For example, if block $\mathrm{ABC}$ was selected randomly, the first patient would be assigned to acupressure group and the second and third patients would be assigned to sham, and control groups, respectively and so on.) Assignment of patients to groups was continued until the completion of the number of participants.

\subsection{Assigning and Blinding}

A total of 90 patients were randomly assigned to three groups of acupressure $(n=30)$, sham $(n=30)$, and controls $(n=30)$ groups. Regarding the acupressure group and during the study, 3 participants were excluded due to intolerance (1 person) and early discharge ( 2 people) in the third and seventh days of the intervention. Because of noncooperation, 2 patients were excluded from the sham group. At the end, the acupressure group $(n=27)$, sham group $(n=28)$, and control group $(n=30)$, with a total of 85 patients were analyzed (Figure 2 ). In order to avoid bias in performing acupressure, the outcome of blinding was assessed by an assistant who was blinded to the type of treatment. Therefore, acupressure was performed by the researcher in two groups of intervention and sham, and the results were evaluated independently by the assistant using STAI.

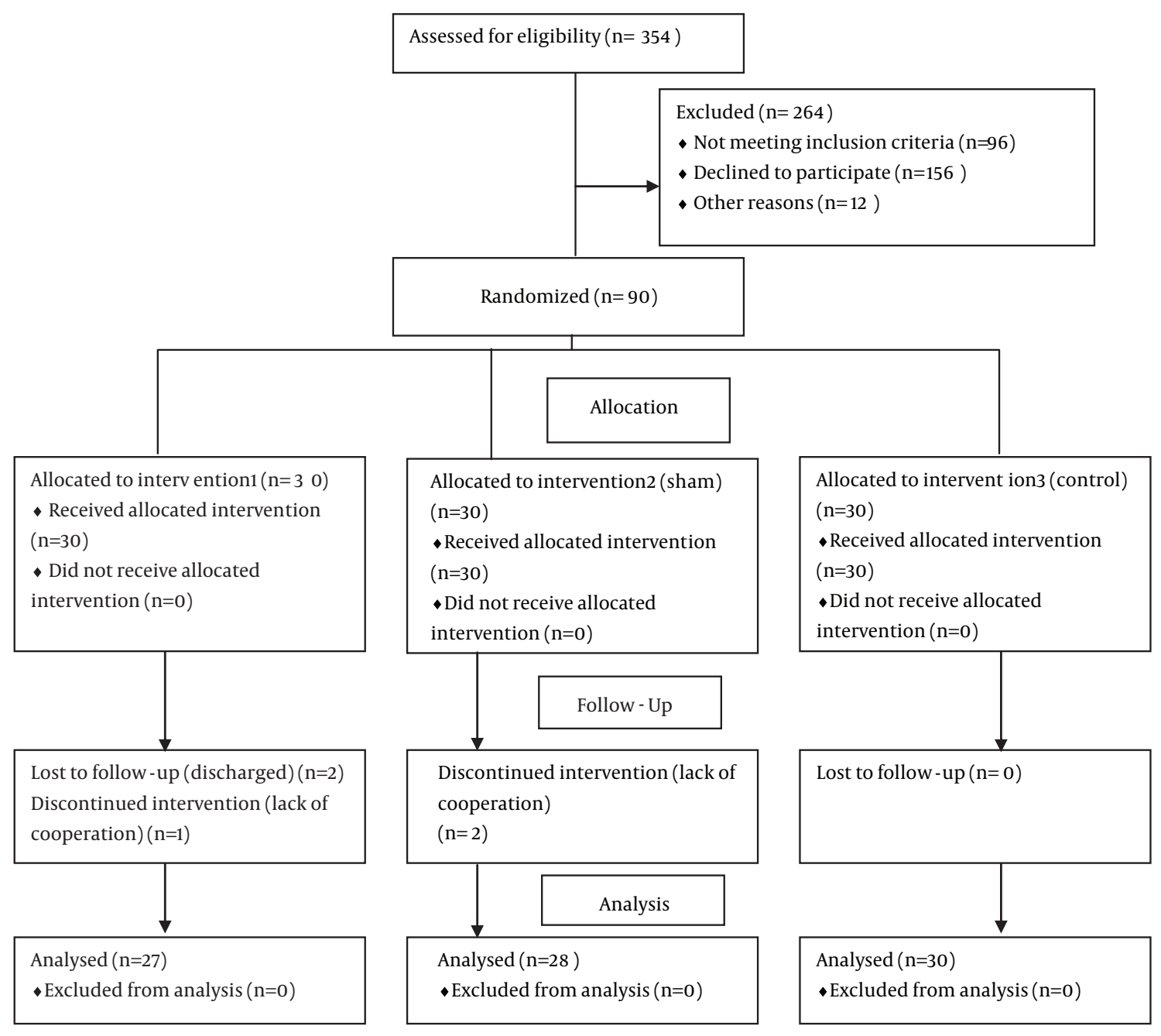

Figure 2. CONSORT Diagram of Flow of Participants Through the Clinical Trial 
Beikmoradi A et al.

\subsection{Statistical Analyze}

After data collection, extraction, and entry into the computer using SPSS for Windows (version 16; SPSS Inc., Chicago, IL, USA), the data were statistically analyzed. Analysis of variance (ANOVA) and chi-square test were performed to compare the qualitative and quantitative variables. In order to compare the means, ANOVA with repeated measures was performed. Moreover, for repeated measurements of traits, least significant difference (LSD), and post-hoc test were used. The significance level of tests was considered at $5 \%$.

\section{Results}

In this study, state and trait anxiety were evaluated before and after the intervention in 3 groups of acupressure, sham, and control. Among the participating patients with cancer, 54.12\% were male and others were female. The mean age and standard deviation of the studied patients with cancer was $49 \pm 12$ years. Moreover, $87 \%$ of the patients were married, $60 \%$ of them lived in the city, and $48.63 \%$ of them had $3-5$ children. In addition, $50.59 \%$ of the patients were in the stage 4 of the disease, $45.88 \%$ of the patients had a history of surgery and chemotherapy, and $91.73 \%$ of them did not use anti-anxiety drugs. Chi-square test and analysis of variance showed that there was no significant difference among 3 groups of acupressure, sham, and control regarding the demographic characteristics. ANOVA test before the intervention showed that there was no significant difference between total score of anxiety (state and trait) of patients with cancer in the study groups $(\mathrm{P}=0.45, \mathrm{~F}=0.81, \mathrm{P}=0.254, \mathrm{~F}=1.393)$. Based on the results of the abovementioned tests, these 3 groups were homogeneous (Table 1).

Table 1. Demographic Characteristics of Patients With Cancer Before the Intervention ${ }^{a}$

\begin{tabular}{|c|c|c|c|c|c|}
\hline $\begin{array}{l}\text { Demographic } \\
\text { Characteristics }\end{array}$ & $\begin{array}{l}\text { Acupressure } \\
\text { Group }\end{array}$ & Sham Group & Control Group & Total & Test, P Value \\
\hline Age, $y$ & $50 \pm 13$ & $48 \pm 13$ & $49 \pm 10$ & $85(100)$ & $\mathrm{P}=0.82$ \\
\hline Gender & & & & & Chi-square $=0.33, \mathrm{P}=0.85$ \\
\hline Male & $15(55.6)$ & $16(57.1)$ & $15(50)$ & $46(54.23)$ & \\
\hline Female & $12(44.7)$ & $12(42.9)$ & $15(50)$ & $39(45.87)$ & \\
\hline Marital status & & & & & Chi-square $=3.20, P=0.52$ \\
\hline Single & $2(7.4)$ & $3(10.7)$ & $1(3.3)$ & $6(7.13)$ & \\
\hline Married & $22(81.5)$ & $24(85 / 7)$ & $28(93.3)$ & $74(86.83)$ & \\
\hline Divorce & $3(11.1)$ & $1(3.6)$ & $1(3.3)$ & $5(6)$ & \\
\hline Children & & & & & $\mathrm{F}=0.02, \mathrm{P}=0.98$ \\
\hline 0 & $2(7.4)$ & $3(10.8)$ & $2(6.7)$ & $7(8.3)$ & \\
\hline $1-2$ & $5(18.5)$ & $7(25.2)$ & $9(29.7)$ & $21(24.47)$ & \\
\hline 3-5 & $16(59.2)$ & $14(50)$ & $11(36.7)$ & $41(48.63)$ & \\
\hline$>5$ & $4(14.8)$ & $4(14.3)$ & $8(26.7)$ & $16(18.6)$ & \\
\hline Residence & & & & & Chi-square, $=0.72, \mathrm{P}=0.7$ \\
\hline Urban & $17(63)$ & $15(53.6)$ & $19(63.3)$ & $51(59.97)$ & \\
\hline Rural & $10(37)$ & $13(46.4)$ & $11(36.7)$ & $34(40.03)$ & \\
\hline Stage & & & & & Chi-square $=0.16, P=0.1$ \\
\hline 2 & $3(11.1)$ & $3(10.7)$ & $3(10)$ & $9(10.6)$ & \\
\hline 3 & $11(40.7)$ & $11(39.3)$ & $11(36.7)$ & $33(38.9)$ & \\
\hline 4 & $13(48.1)$ & $14(50)$ & $16(53.3)$ & $43(50.47)$ & \\
\hline Previous treatment & & & & & Chi-square $=2, P=0.92$ \\
\hline Chemotherapy & $9(33.3)$ & $12(42.8)$ & $12(40)$ & $33(38.7)$ & \\
\hline Chemosurgery & $14(51.9)$ & $11(39.3)$ & $14(46.2)$ & $39(45.8)$ & \\
\hline Chemoradiotherapy & $1(3.7)$ & $1(3.6)$ & $2(6.7)$ & $4(4.67)$ & \\
\hline $\begin{array}{l}\text { Chemotherapy surgery } \\
\text { radiotherapy }\end{array}$ & $3(11.1)$ & $4(14.3)$ & $2(6.7)$ & $9(10.7)$ & \\
\hline Ant-anxiety drug & & & & & Chi-square $=0.35, P=0.84$ \\
\hline Received & $2(7.4)$ & $3(10.7)$ & $2(6.7)$ & $7(8.27)$ & \\
\hline Unreceived & $25(92.6)$ & $25(89.3)$ & $28(93.3)$ & $78(91.73)$ & \\
\hline
\end{tabular}

\footnotetext{
a Data are presented as mean \pm SD or No. (\%).
} 
Beikmoradi A et al.

The mean state anxiety score in the acupressure group before the intervention was $49.41 \pm 7.07$, and after applying acupressure, it decreased in session 5 to $45.30 \pm 7.14$ and in session 10 to $43.48 \pm 6.82$. ANOVA test showed that the changes in the mean state anxiety score were significant across 3 measurements $(\mathrm{P}=0.001, \mathrm{~F}=9.05)$, with a decreasing trend. Mean state anxiety score in the sham group before the intervention was $48 \pm 8.09$, and after applying sham acupressure, it slightly decreased in the 5th session to $47.57 \pm 7.85$ and in the 10 th session to 46.71 \pm 7.32 . ANOVA test showed that the changes in the mean state anxiety score were not significant across 3 measurements $(\mathrm{P}=0.5, \mathrm{~F}=0.7)$ and had a slight descending trend. Mean state anxiety score in the control group before the intervention was $50.27 \pm 11.49$, and it increased after 5 days of the intervention to $52.13 \pm 12.37$ and after 10 days to $53.50 \pm 10.82$. ANOVA test showed that the changes in the mean state anxiety score were not significant across 3 measurements $(\mathrm{P}=0.76, \mathrm{~F}=0.28$ ) (Table 2 ). Repeated measures ANOVA showed that acupressure had a significant effect on the mean score of state anxiety of the studied patients with cancer $(\mathrm{P}=0.002, \mathrm{~F}=6.73, \mathrm{df}$ $=2$ ). LSD test showed a significant difference between mean state anxiety scores of acupressure and control groups $(\mathrm{P}<0.001)$ and that of sham and control groups
$(P=0.009)$. Nevertheless, there was no significant difference between mean state anxiety scores of acupressure and sham groups $(\mathrm{P}=0.43)$ (Table 3$)$.

The mean trait anxiety score of the patients in the acupressure group before the intervention was $46.89 \pm 7.66$. There was a slight decrease in this score after the acupressure in the $5^{\text {th }}$ session $(45.48 \pm 7.92)$ and on the $10^{\text {th }}$ session $(45.63 \pm 8.08)$. ANOVA test showed that the changes in mean trait anxiety score were not significant across the 3 measurements $(\mathrm{P}=0.08, \mathrm{~F}=2.66)$. The mean trait anxiety score of patients in the sham group before the intervention was $46.68 \pm 7.87$, and after acupressure, it increased to $47.96 \pm 6.33$ in the $5^{\text {th }}$ session and in the $10^{\text {th }}$ session reached to $46.89 \pm 6.94$. ANOVA test showed that the changes in mean trait anxiety score were not significant across the 3 measurements $(P=0.59, F=0.53)$. The mean trait anxiety score of patients in the control group before the intervention was $45.80 \pm 10.66$. This score increased after 5 days of the study to $46.90 \pm 10.30$ and on the $10^{\text {th }}$ day to $46.80 \pm 8.96$. ANOVA test showed that the changes in mean trait anxiety score were not significant across 3 measurements $(\mathrm{P}=0.15, \mathrm{~F}=1.98)$. Furthermore, ANOVA with repeated measures showed that acupressure did not have a significant effect on mean anxiety of participants $(\mathrm{P}=0.77, \mathrm{~F}=0.26, \mathrm{df}=2)$ (Table 4$)$.

\begin{tabular}{|c|c|c|}
\hline & State Anxiety Score & ANOVA \\
\hline Acupressure group $(n=27)$ & & $P=0.01, F=9.05$ \\
\hline Before intervention & $49.41 \pm 7.07$ & \\
\hline After $5^{\text {th }}$ session & $45.30 \pm 7.14$ & \\
\hline After $10^{\text {th }}$ session & $43.48 \pm 6.82$ & \\
\hline Sham group $(n=28)$ & & $\mathrm{P}=0.5, \mathrm{~F}=0.7$ \\
\hline Before intervention & $48 \pm 8.09$ & \\
\hline After $5^{\text {th }}$ session & $47.57 \pm 7.85$ & \\
\hline After $10^{\text {th }}$ session & $46.71 \pm 7.32$ & \\
\hline Control group $(\mathbf{n}=\mathbf{3 0})$ & & $\mathrm{P}=0.76, \mathrm{~F}=0.28$ \\
\hline Before intervention & $50.27 \pm 11.49$ & \\
\hline After $5^{\text {th }}$ session & $52.13 \pm 12.37$ & \\
\hline After $10^{\text {th }}$ session & $53.50 \pm 10.82$ & \\
\hline
\end{tabular}

${ }^{\mathrm{a}}$ Data are presented as mean $\pm \mathrm{SD}$.

Table 3. LSD Test

\begin{tabular}{lcc}
\hline Group & Mean Difference \pm SE & PValue \\
\hline Acupressure-control & $-5.90 \pm 1.70$ & 0.001 \\
Acupressure-sham & $-1.37 \pm 1.73$ & 0.43 \\
Sham- control & $-4.54 \pm 1.68$ & 0.009 \\
\hline
\end{tabular}


Beikmoradi A et al.

\begin{tabular}{|c|c|c|}
\hline & Trait Anxiety Score & ANOVA \\
\hline Acupressure group $(n=27)$ & & $\mathrm{P}=0.08, \mathrm{~F}=2.66$ \\
\hline Before intervention & $46.89 \pm 7.66$ & \\
\hline After $5^{\text {th }}$ session & $45.48 \pm 7.92$ & \\
\hline After $10^{\text {th }}$ session & $45.63 \pm 8.08$ & \\
\hline Sham group $(n=28)$ & & $\mathrm{P}=0.59, \mathrm{~F}=0.53$ \\
\hline Before intervention & $46.68 \pm 7.87$ & \\
\hline After $5^{\text {th }}$ session & $47.96 \pm 6.33$ & \\
\hline After $10^{\text {th }}$ session & $46.89 \pm 6.94$ & \\
\hline Control group $(n=30)$ & & $\mathrm{P}=0.15, \mathrm{~F}=1.98$ \\
\hline Before intervention & $45.80 \pm 10.66$ & \\
\hline After $5^{\text {th }}$ session & $46.90 \pm 10.30$ & \\
\hline After $10^{\text {th }}$ session & $46.80 \pm 8.96$ & \\
\hline
\end{tabular}

\section{Discussion}

The results of this study showed that performing acupressure significantly decreased mean score of state anxiety in the acupressure group. While performing fake acupressure in sham and control groups did not significantly affect the mean anxiety score. Therefore, acupressure had an influence on the state anxiety level. The statistical tests also showed significant changes in state anxiety mean score in sham and control groups. This showed the effect of placebo acupressure on anxiety in patients with cancer. With regard to the strong points of our study, this was the first clinical trial on the effects of acupressure on the anxiety of patients with cancer, which also showed significant improvement of mean score within a period of 10 days. Acupressure may stimulate endogenous opioid system, and thus, affects intermediate behaviors and facilitates psychological improvement. The features of this study were the daily application of acupressure and the long duration of each session, which were the other strong points of this study. If acupressure intervention was performed more frequently and the treatment duration was longer, perhaps better results would be obtained. In this study, because of the possible discharge of the patients during the treatment period, the determined duration of the treatment was 10 days, which was the weak point of this study.

In the present study, by performing acupressure, the mean state anxiety score in the intervention group significantly decreased. The results of the study by MichalekSauberer et al. (36) on the effect of ear acupuncture on dental anxiety of patients before treatment showed decrease of state anxiety score immediately before dental treatment in the ear acupuncture group $(\mathrm{P}<0.001)$. Results of the studies of Rosted et al. (37), Wu et al. (38), and Boucher et al. (39) on the effect of acupuncture on the anxiety of patients showed the beneficial impact of this intervention in reducing anxiety in the experimental group. The study conducted by Bussell et al. (40), which examined the effect of acupuncture on anxiety and memory functioning of students, showed that those who received acupuncture had less anxiety after the intervention compared to the control group. The results of the study by Isoyama et al. (41), who examined the effect of acupuncture on anxiety symptoms in women undergoing in vitro fertilization, showed a significant decrease in mean Hamilton anxiety score in the experimental group in comparison to the control group. In other study, researchers proposed the effectiveness of acupuncture on patients' anxiety based on STAI. The results of this study showed that the anxiety level of the candidates undergoing outpatient surgery, after ear acupuncture had significantly decreased (15). The results of the study by Valiee et al. (25) on the effect of acupressure on preoperative anxiety showed a statistically significant difference in the anxiety of the experimental group before and immediately after the intervention $(\mathrm{P}<0.001)$. The results of all these studies are consistent with the results of the present study. The study by Kao et al. (23) on the effect of ear acupressure on anxiety in premenopausal women and at the onset of menopause had no evidence to support that ear acupuncture was better in relieving anxiety compared to sham group. In the present study, this inconsistency may be explained due to using most of the effective acupoints on anxiety, daily interventions, and performing it by an acupressure therapist.

In the present study, the statistical tests showed significant changes in the mean state anxiety score in the sham and control groups. However, the changes in the mean state anxiety score of the intervention group and sham group were not significant. This showed the effect of placebo sham acupressure on the patients with cancer. Tsay 
Beikmoradi A et al.

et al. (34) studied the effect of acupressure on fatigue in dialysis patients and showed a statistically significant difference between the fatigue score of acupressure and control groups $(\mathrm{P}<0.001)$ and also between sham and control groups $(\mathrm{P}<0.001)$. In general, the impact of inculcation was reported for acupuncture and acupressure techniques, which we attempted to neutralize through sham group. Nevertheless, in most cases, positive changes also occur in the sham group. Thus, small changes in the scores of sham group might be due to the psychological and physiological effects of touch, which cause various comfort levels in the body, which alleviates anxiety to some extent (25). This decrease in anxiety might be due to their expectation (of the effectiveness of the study), feeling safe (because of the presence of researcher as a member of the medical team), or the effect of their awareness of being studied (34).

The study by Boucher et al. on the effect of acupuncture on students exam anxiety, also showed significant changes in the sham acupuncture group ( $P=0.01)(39)$. The study by Valiee et al. (25) also showed statistically significant differences in anxiety before and immediately after the intervention in the placebo group, which is inconsistent with the present study. This difference might be due to the different population of this study, and the chronic nature of cancer and its accompanying high anxiety levels. In the study by Molassiotis et al. (42), there was no clear placebo effect or improvement was observed in the sham acupressure group. This might be due to the small sample size $(\mathrm{n}=16)$.

In the present study, the mean score of state anxiety improved in the acupressure intervention group by 5.93 scores and in the sham group by 1.29 scores. Moreover, the average decrease in the anxiety level in the acupressure intervention group was 4.64 scores higher than the sham group and 10.02 scores higher than the control group. The study by Michalek-Sauberer et al. (36) on the effect of ear acupuncture on anxiety before dental treatment showed that the anxiety score improved in ear acupuncture group by 7.3 scores and in the sham group by 3.7 scores. In addition, the average decrease in anxiety level in the ear acupuncture group was 3.6 scores higher than that of the sham group and 9.9 scores higher than that of the control group. This result was in accordance with the present study.

The present study showed that the mean trait anxiety score in the experimental group after the intervention had a slight decrease, and in the sham and control groups, it had a slight increase, which was not statistically significant. The results of the study by Acar et al. (43) examining the effect of acupuncture on patients' preoperative anxiety showed no change in the anxiety score of patients in the acupuncture and sham groups.

The study by Mousavi et al. (44) on the effect of acupressure on the anxiety of nursing and midwifery students, as well as operating room technicians at the beginning of their training showed significant reduction of anxiety.
This was inconsistent with the present study because in this study the anxiety score decreased in the experimental group, however, the difference was not significant. This study was conducted on healthy participants with lower anxiety levels than the patients with cancer. Apparently, in this study the sample size (30 patients) was small, and it would have been better if more people had participated. In the study by Mousavi et al. (45) on the effect of acupressure on pre-amputation anxiety, anxiety levels reduced from $76 \%$ to $67 \%$, which confirmed the effectiveness of acupressure on reducing anxiety levels of the participants. This was not consistent with the present study; the difference in acupoints used and the number of sessions were the reasons for this difference.

Results of the current study showed that acupressure was effective on the anxiety of patients with cancer. Considering the high prevalence of anxiety and its symptoms in cancerous patients, this study suggests using acupressure as a nonpharmacological treatment. This study provides a bright prospect for using complementary and alternative medicine, especially acupressure, to relieve patients' anxiety by their nurses, to decrease their health problems, to improve their quality of life. Furthermore, it can be easily performed by the patients themselves.

It is recommended for future studies to carry out this research with 3 groups and for a longer duration. Because of the different types of cancer in this study, future studies on acupressure should be investigated on one specific type of cancer. Moreover, the effect of acupressure should be compared with other anxiety reducing medications as well as other complementary treatment methods. Finally, the effect of acupressure on other types of chronic diseases should also be investigated.

\section{Acknowledgements}

Our appreciation goes to all nurses of the hematology ward of Shahid Beheshti Hospital in Hamadan, as well as patients and their families who helped us in performing this study, and finally all officials involved in the approval and implementation of this research.

\section{Authors' Contributions}

Ali Beikmoradi and Fatemeh Najafi: contributed to all research processes, study concept and design, acquisition of data, and drafting of the manuscript. Ghodratallah Roshanaei: analysis and interpretation of data. Zahra Pour Esmaeil and Alireza Ahmadi: technical and material support. Mahnaz Khatibian: drafting of the manuscript.

\section{Funding/Support}

This article was part of a nursing thesis that was funded by Hamadan University of Medical Sciences, IR Iran.

\section{References}

1. Petersen PE. Oral cancer prevention and control--the approach of the World Health Organization. Oral Oncol. 2009;45(4-5):454-60. 
Beikmoradi A et al.

2. Malekian A, Alizadeh A, Ahmadzadeh GH. Anxiety and depression in cancer patients. J Res Behav Sci. 2008;5(2):115-9.

3. Spencer R, Nilsson M, Wright A, Pirl W, Prigerson H. Anxiety disorders in advanced cancer patients: correlates and predictors of end-of-life outcomes. Cancer. 2010;116(7):1810-9.

4. Teunissen SC, de Graeff A, Voest EE, de Haes IC. Are anxiety and depressed mood related to physical symptom burden? A study in hospitalized advanced cancer patients. Palliat Med. 2007;21(4):341-6.

5. Miovic M, Block S. Psychiatric disorders in advanced cancer. Cancer. 2007;110(8):1665-76.

6. Stark D, Kiely M, Smith A, Velikova G, House A, Selby P. Anxiety disorders in cancer patients: their nature, associations, and relation to quality of life. J Clin Oncol. 2002;20(14):3137-48.

7. Garfami H, Shafi Abadi A, Sanaee Zaker B. Effectiveness of group logo therapy in reducing mental symptoms of breast cancer patients. Andisheh Raftar (Appl Psychol). 2009;4(13):35-42.

8. Noghani F, Monjamed Z, Bahrani N, Ghodrati Jablo V. The comparison of self-esteem between male and female cancer patients. Hayat. 2006;12(2):33-41.

9. White L. Foundations of nursing.: Delmar Pub;2004

10. Arrieta O, Angulo LP, Nunez-Valencia C, Dorantes-Gallareta Y, Macedo EO, Martinez-Lopez D, et al. Association of depression and anxiety on quality of life, treatment adherence, and prognosis in patients with advanced non-small cell lung cancer. Ann Surg Oncol. 2013;20(6):1941-8.

11. Lim CC, Devi MK, Ang E. Anxiety in women with breast cancer undergoing treatment: a systematic review. Int J Evid Based Healthc. 2011;9(3):215-35

12. Khan NF, Ward AM, Watson E, Rose PW. Consulting and prescribing behaviour for anxiety and depression in long-term survivors of cancer in the UK. Eur J Cancer. 2010;46(18):3339-44.

13. Zhang M, Liu X, Li J, He L, Tripathy D. Chinese medicinal herbs to treat the side-effects of chemotherapy in breast cancer patients. Cochrane Database Syst Rev. 2007(2):CD004921.

14. Karst M, Winterhalter M, Munte S, Francki B, Hondronikos A, Eck$\operatorname{ardt} \mathrm{A}$, et al. Auricular acupuncture for dental anxiety: a randomized controlled trial. Anesth Analg. 2007;104(2):295-300.

15. Pilkington K. Anxiety, depression and acupuncture: A review of the clinical research. Auton Neurosci. 2010;157(1-2):91-5.

16. Cherry B, Jacob SR. Contemporary Nursing, Issues, Trends, and Management. 6th ed: Elsevier Health Sciences; 2013

17. Shen J, Andersen R, Albert PS, Wenger N, Glaspy J, Cole M, et al. Use of complementary/alternative therapies by women with advanced-stage breast cancer. BMC Complement Altern Med. 2002:2:8

18. Lee EJ, Frazier SK. The efficacy of acupressure for symptom management: a systematic review. J Pain Symptom Manage. 2011; 42(4):589-603.

19. Zick SM, Alrawi S, Merel G, Burris B, Sen A, Litzinger A, et al. Relaxation acupressure reduces persistent cancer-related fatigue. Evid Based Complement Alternat Med. 2011;2011.

20. Wu HS, Wu SC, Lin JG, Lin LC. Effectiveness of acupressure in improving dyspnoea in chronic obstructive pulmonary disease. $J$ Adv Nurs. 2004;45(3):252-9.

21. Ouyang H, Chen JD. Review article: therapeutic roles of acupuncture in functional gastrointestinal disorders. Aliment Pharmacol Ther. 2004;20(8):831-41.

22. Yang $\mathrm{CH}$, Lee $\mathrm{BH}$, Sohn $\mathrm{SH}$. A possible mechanism underlying the effectiveness of acupuncture in the treatment of drug addiction. Evid Based Complement Alternat Med. 2008;5(3):257-66.

23. Kao CL, Chen CH, Lin WY, Chiao YC, Hsieh CL. Effect of auricular acupressure on peri- and early postmenopausal women with anxiety: a double-blinded, randomized, and controlled pilot study. Evid Based Complement Alternat Med. 2012;2012:567639.

24. Maa SH, Tsou TS, Wang KY, Wang CH, Lin HC, Huang YH. Self-administered acupressure reduces the symptoms that limit daily activities in bronchiectasis patients: pilot study findings. J Clin Nurs. 2007;16(4):794-804
25. Valiee S, Bassampour SS, Nasrabadi AN, Pouresmaeil Z, Mehran A. Effect of acupressure on preoperative anxiety: a clinical trial. $J$ Perianesth Nurs. 2012;27(4):259-66.

26. Agarwal A, Ranjan R, Dhiraaj S, Lakra A, Kumar M, Singh U. Acupressure for prevention of pre-operative anxiety: a prospective, randomised, placebo controlled study. Anaesthesia. 2005;60(10):978-81.

27. Kober A, Scheck T, Schubert B, Strasser H, Gustorff B, Bertalanffy P, et al. Auricular acupressure as a treatment for anxiety in prehospital transport settings. Anesthesiology. 2003;98(6):1328-32.

28. Barker R, Kober A, Hoerauf K, Latzke D, Adel S, Kain ZN, et al. Out-of-hospital auricular acupressure in elder patients with hip fracture: a randomized double-blinded trial. Acad Emerg Med. 2006;13(1):19-23.

29. Mousavi SM, Alamolhoda AA, Gouya MM, Lickiss N. Implementation of Comprehensive National Cancer Control Program in Iran: an experience in a developing country. Ann Oncol. 2008; 19(2):398-400.

30. Julian LJ. Measures of anxiety: State-Trait Anxiety Inventory (STAI), Beck Anxiety Inventory (BAI), and Hospital Anxiety and Depression Scale-Anxiety (HADS-A). Arthritis Care Res (Hoboken). 2011;63 Suppl 11:S467-72.

31. Kvaal K, Ulstein I, Nordhus IH, Engedal K. The Spielberger State-Trait Anxiety Inventory (STAI): the state scale in detecting mental disorders in geriatric patients. Int J Geriatr Psychiatry. 2005;20(7):629-34

32. Reza H, Kian N, Pouresmail Z, Masood K, Sadat Seyed Bagher M Cheraghi MA. The effect of acupressure on quality of sleep in Iranian elderly nursing home residents. Complement Ther Clin Pract. 2010;16(2):81-5.

33. Stux G, Berman B, Pomeranz B. Basics of Acupuncture.NewYork: Springer; 2003.

34. Tsay SL. Acupressure and fatigue in patients with end-stage renal disease-a randomized controlled trial. Int J Nurs Stud. 2004;41(1):99-106.

35. Norman GR, Streiner DL. Biostatistics: the bare essentials.Bc Decker Hamilton: Ontario; 2008

36. Michalek-Sauberer A, Gusenleitner E, Gleiss A, Tepper G, Deusch E. Auricular acupuncture effectively reduces state anxiety before dental treatment--a randomised controlled trial. Clin Oral Investig. 2012;16(6):1517-22.

37. Rosted P, Bundgaard M, Gordon S, Pedersen AM. Acupuncture in the management of anxiety related to dental treatment: a case series. Acupunct Med. 2010;28(1):3-5.

38. Wu S, Liang J, Zhu X, Liu X, Miao D. Comparing the treatment effectiveness of body acupuncture and auricular acupuncture in preoperative anxiety treatment. J Res Med Sci. 2011;16(1):39-42.

39. Boucher C, Griffith L, Siepler D, Tilley L. The Effect of Traditiona Chinese Medical Acupuncture (TCM) on Test Anxiety (A Pilot Study): Logan University; 2011.

40. Bussell J. The effect of acupuncture on working memory and anxiety. J Acupunct Meridian Stud. 2013;6(5):241-6.

41. Isoyama D, Cordts EB, de Souza van Niewegen AM, de Almeida Pereira de Carvalho W, Matsumura ST, Barbosa CP. Effect of acupuncture on symptoms of anxiety in women undergoing in vitro fertilisation: a prospective randomised controlled study. Acupunct Med.2012;30(2):85-8.

42. Molassiotis A, Sylt P, Diggins $H$. The management of cancerrelated fatigue after chemotherapy with acupuncture and acupressure: a randomised controlled trial. Complement Ther Med. 2007;15(4):228-37.

43. Acar HV, Cuvas O, Ceyhan A, Dikmen B. Acupuncture on Yintang point decreases preoperative anxiety. J Altern Complement Med. 2013;19(5):420-4

44. Mousavi S, Mirzaei M, Reza Soltani P. The Effects of Acupressure on Anxiety Nursing, Midwifery and Operating Room Students. J Guilan Univ Med Sci. 2009;18(71):82-9.

45. Mousavi S. Acupressure and anxity in patients before amputation in poorsina hospital-Rasht. Iran J Surg. 2007;15(1):101-7. 